\title{
Old Is (Not) Gold: Midazolam Monotherapy versus Midazolam Plus Fentanyl for Sedation during Cardiac Catheterization
}

\author{
William Black $\mathbb{D}^{1},{ }^{1}$ Raj Baljepally $\mathbb{D}^{1},{ }^{1}$ Laylan Shali, ${ }^{1}$ Omar Alsharif, ${ }^{1}$ Scott Warden, ${ }^{1}$ \\ Eric Heidel, ${ }^{1}$ and Xiaopeng Zhao $\mathbb{1}^{2}$ \\ ${ }^{1}$ University of Tennessee Medical Center, Heart Lung Vascular Institute, Department of Cardiology, Knoxville, TN, USA \\ ${ }^{2}$ Department of Mechanical, Aerospace, and Biomedical Engineering, University of Tennessee, Knoxville, TN, USA \\ Correspondence should be addressed to William Black; wblack@utmck.edu
}

Received 10 March 2021; Revised 16 July 2021; Accepted 25 July 2021; Published 3 August 2021

Academic Editor: Shenghua Zhou

Copyright (C) 2021 William Black et al. This is an open access article distributed under the Creative Commons Attribution License, which permits unrestricted use, distribution, and reproduction in any medium, provided the original work is properly cited.

\begin{abstract}
Objective. We aimed to study the differences in perception of pain during cardiac catheterization with midazolam monotherapy compared to the current standard of midazolam plus fentanyl. Background. Procedural sedation is important to ensure comfort and safety in patients undergoing left heart catheterization. Despite the widespread use of midazolam and fentanyl for procedural sedation, the effectiveness of this dual agent approach to sedation has never been studied in comparison to midazolam monotherapy. Methods. A total of 129 patients undergoing sedation for outpatient elective cardiac catheterization were randomly assigned to either midazolam monotherapy $(n=69)$ or combination of midazolam and fentanyl $(n=60)$. The primary outcome was assessment of pain perception prior to discharge by patient completion of a pain questionnaire. Participants were asked if they experienced any pain during their procedure (yes/no) and, if yes, asked to rate their overall pain level using a 10-point Likert scale that ranged from 1 (minimal pain) to 10 (worst pain imaginable). Results. Most patients $(n=94,73 \%)$ reported no pain during their procedure. Patients sedated with midazolam monotherapy reported similar average pain scores compared to patients sedated with the combination of midazolam and fentanyl (1.1 vs. 1.1, $p=0.95)$. Conclusions. Among patients undergoing elective cardiac catheterization, no significant differences in pain scores were noted between sedation with midazolam alone compared to midazolam and fentanyl. Due to fentanyl's unfavorable interaction with P2Y12 agents, increased costs, and addiction potential, it is imperative that cardiologists revisit the role of effective procedural sedation with a single agent and avoid the use of fentanyl.
\end{abstract}

\section{Introduction}

The administration of procedural sedation is a common practice to promote safety and ensure patient comfort during cardiac catheterization procedures $[1,2]$. The Society for Cardiovascular Angiography and Interventions expert consensus statement on best practices in the cardiac catheterization laboratory encourages the use of moderate sedation for patients undergoing coronary angiography [1]. Intravenous midazolam and fentanyl have been used historically to achieve adequate sedation for cardiac catheterization procedures, and this dual therapy has remained the mainstay of procedural sedation in the cardiac cath lab as well as the electrophysiology lab [3-6]. Despite the widespread use of this practice, there is little clinical evidence evaluating the effectiveness of these medications for patients undergoing catheterization procedures. The rate of sedation usage for cardiac procedures varies widely and may be influenced by culture, patient expectations, training, and geography [7]. An international survey found that $92 \%$ of cardiologists in North America reported using sedation during cardiac catheterization compared to just $38 \%$ of cardiologists in other countries [8]. The same study found that a majority of US cardiologists $(60 \%)$ perceived that $75-100 \%$ of their patients would want sedation, while a majority of 
European cardiologists (60\%) perceived that only 0-25\% of their patients would want sedation.

Opioids have been the cornerstone of analgesia for both chronic and acute pain since the late 1980s as part of a push by the Joint Commission to label pain as "the fifth vital sign" [9]. The rise in opioid addiction is an unintended consequence of this campaign and is now a major public health crisis facing the United States [10, 11]. In 2016 alone, more than 42,0000 Americans died from opioid overdose, and the annual number of opioid deaths is projected to reach nearly 82,000 by 2025 $[12,13]$. There is mounting evidence that the risk of opioid dependence increases with even a single opioid exposure, and many heroin addicts were first exposed to opioids by a legitimate medical prescription $[14,15]$. A recent retrospective cohort study found that opioidnaive patients who were prescribed opioids in the emergency department for acute pain are at an increased risk for additional opioid use at one year compared to patients who were not prescribed opioids [16, 17]. Despite the potential for postprocedural opioid use, preprocedural screening for a prior history of substance abuse is not routinely performed by cardiologists prior to catheterization [18].

In addition to the potential for opioid abuse, the use of opioid medications may interfere with the absorption of P2Y12 inhibitors due to delayed gastric emptying. Rapid absorption of P2Y12 inhibitors is critical for patients with acute coronary syndrome and patients undergoing percutaneous coronary intervention to prevent acute stent thrombosis $[19,20]$. The PACIFY trial demonstrated that fentanyl delayed the absorption of ticagrelor by up to four hours and resulted in attenuated platelet inhibition at two hours [21]. Interestingly, there was no significant difference in average patient comfort between the fentanyl and nofentanyl arms in that study (all patients received local anesthetic and midazolam).

Several small studies have demonstrated adequate sedation for catheter-based procedures with either minimal sedation or benzodiazepine monotherapy [22-24]. The combination of benzodiazepines and opioids has been shown to reduce the risk of radial artery spasm and improve patient tolerability [25]. Intraarterial vasodilators such as nitroglycerin and verapamil are routinely administered to reduce radial artery spasm, so it is unclear whether the addition of fentanyl is necessary to prevent radial artery spasm or if sedation with benzodiazepines alone would be adequate. We sought to compare the differences in pain perception with midazolam alone versus the current standard of care of midazolam plus fentanyl during cardiac catheterization.

\section{Methods}

2.1. Study Design. This is a single-center, single-blinded randomized prospective study performed at the University of Tennessee Medical Center, Knoxville, TN, USA. The study was approved by the institutional review board (IRB).
2.2. Study Population. Patients aged 18 years and older who were referred for outpatient elective cardiac catheterization (CC) including left heart catheterization (LHC), right heart catheterization (RHC), and LHC with coronary angiography from July 2019 to September 2020 were eligible for inclusion in the study. Patients were excluded if they had a known allergy to one or both of the study medications.

2.3. Procedural Details. Informed consent was obtained, and patients were randomly assigned to two groups: midazolam monotherapy $(n=69)$ and midazolam plus fentanyl $(n=60)$. The randomization sequence was computer generated and concealed in sealed opaque envelopes. Patients, but not CC operators, were blinded to the study medications. Patients in the midazolam group were given intravenous midazolam with dosages based on age, gender, and body mass (0.5-4 mg) in accordance with normal pharmacological practice. Patients in the midazolam and fentanyl groups were given intravenous midazolam $(0.5-4 \mathrm{mg})$ plus intravenous fentanyl (25-125 mcg) with fentanyl similarly dosed based on age, gender, and body mass. Patients from the midazolam group that required additional sedation were given a second dose of midazolam. If adequate sedation could not be achieved with midazolam alone, they were allowed to crossover and receive fentanyl. In all patients, local anesthetic ( $1 \%$ xylocaine) was administered at the access site after administration of sedation. Oral diazepam (5-10 mg) and diphenhydramine $(25 \mathrm{mg}$ ) were given 30 minutes before commencement of the procedure unless patients were allergic to these medications. After the procedure, patients who underwent femoral arterial access were positioned supine and flat for at least 2 hours (longer if no closure device was deployed). Patients who underwent catheterization via the right radial artery had a radial compression bracelet placed and were allowed to sit up after returning to the recovery room. Patients were administered a pain survey by a research assistant to complete at the time of discharge ( 3 hours after the completion of the procedure for radial access and 6 hours after the procedure for femoral access).

Participants were asked if they experienced any pain during their procedure (yes/no) and, if yes, asked to rate their overall pain level for the periprocedural period using a 10-point Likert scale that ranged from 1 (minimal pain) to 10 (worst pain imaginable). Patients that had undergone prior LHC were asked to compare their level of pain to prior LHC (less pain, the same, or more pain).

2.4. Statistical Analysis. We hypothesized a medium effect size, $d=0.5$, associated with the difference in treatment modalities. Using the aforementioned effect size, a two-sided hypothesis, an alpha value of 0.05 , a beta value of 0.20 , and an equal allocation ratio to treatment arms $(1: 1)$, it was found that $n=128$ participants would be needed in the study to achieve adequate statistical power to detect a difference if it actually existed in the population. The sample size calculation was performed using $\mathrm{G} *$ Power Version 3.1. 
Statistical analysis was performed using chi-square analyses to compare the midazolam and midazolam plus fentanyl groups on categorical variables. Frequency and percentage statistics were used to describe the statistical findings of the chi-square statistics. Independent-samples $t$ tests were used to compare the groups on continuous variables. Means and standard deviations were reported and interpreted for the $t$-test findings. Statistical significance was assumed at an alpha value of 0.05 , and all analyses were performed using SPSS Version 26 (Armonk, NY: IBM Corp.).

\section{Results}

A total of 129 patients were enrolled and completed the patient pain questionnaire (Figure 1). Participants aged from 32 to 85 years (mean 65.6, standard deviation 10.8). The majority $(83 \%, n=107)$ were classified as overweight or obese according to their body mass index $\left(\geq 25 \mathrm{~kg} / \mathrm{m}^{2}\right)$. The baseline demographics of the study population are listed in Table 1 . The majority of patients in each group underwent radial access and had LHC performed as indicated.

In general, patients reported a low level of discomfort during the procedure. The percentage of patients who experienced any pain was similar between the midazolam and midazolam plus fentanyl groups $(26.1 \%$ vs. $28.3 \%, p=0.78)$. The average pain scores were identical between the two treatment groups as indicated in Table 2 (1.1 vs. 1.1, $p=0.95)$. Nearly half of the patients had undergone prior LHC $(n=63,49 \%)$. Twenty-seven patients reported similar pain compared to their last LHC, 30 reported less pain, and 6 patients reported more pain, which did not statistically differ between the two groups. Of the 69 patients in the midazolam monotherapy group, only $16 \%(n=11)$ crossed over and received fentanyl.

There were 6 patients in each treatment group who reported a history of chronic pain. Patients with a history of chronic pain who received only midazolam reported higher average pain scores than patients with chronic pain who received both midazolam and fentanyl ( 2.83 vs. 0.5 , $p=0.037)$. Patients who reported pain during their procedure had longer average procedure times compared to patients who did not report any pain (52.51 minutes vs. 35.84 minutes, $p=0.006)$. The rates of pain did not statistically differ based on artery accessed (31.6\% for femoral access, $25.3 \%$ for radial access, $p=0.46 \%)$. Of the patients who did report pain, pain scores did not differ based on arterial access site (1.21 for femoral access, 1.01 for radial access, $p=0.63)$.

\section{Discussion}

Sedation is desirable during catheter-based cardiac procedures to produce analgesia and to improve overall patient comfort [26]. Despite the prevalence of coronary angiography, there is a paucity of data regarding optimal sedation techniques for this procedure. The purpose of this study was to examine the effectiveness of sedation with midazolam alone versus the combination of midazolam and fentanyl in patients undergoing elective CC. Patient comfort was evaluated after the procedures by completion of a pain questionnaire prior to discharge. Most patients reported no pain at all during their procedure. Of the 35 patients who did experience pain during their procedure, 28 reported their pain to be less than 5 on a scale from 1 to 10 . For the primary endpoint of patient reported periprocedural pain, there was no difference in midazolam alone compared to midazolam and fentanyl combination therapy. Our study demonstrates that midazolam monotherapy is an acceptable choice for procedural sedation for CC.

There are clinical scenarios where an initial sedation strategy with midazolam and fentanyl may still be utilized. Although we enrolled few patients with a history of chronic pain $(n=12)$, patients with chronic pain reported lower average pain scores when they received both fentanyl and midazolam for sedation ( 0.5 vs. $2.83, p=0.037)$. Longer cases may benefit from sedation with midazolam and fentanyl as well. Patients who reported pain had an average procedure length of 52.51 minutes ( $\mathrm{SD}=22.87$ minutes) compared to 35.84 minutes (SD 43.91) in patients who did not report pain $(p=0.006)$. Musculoskeletal pain, particularly lumbar pain, is the most common site of pain reported during left heart catheterization [27]. Although we did not ask patients to localize their pain, we suspect that patients with longer procedural times experienced more musculoskeletal pain due to discomfort from the hard cath table. There was no significant difference in average reported pain scores in patients who underwent femoral arterial access compared to radial arterial access $(1.21, \mathrm{SD}=2.01$ vs. 1.01 , $\mathrm{SD}=2.15, p=0.63$ ).

There are several potential benefits to using midazolam monotherapy for procedural sedation. First, it has been welldemonstrated that fentanyl administration lowers plasma concentrations of ticagrelor and delays its antiplatelet effects as demonstrated in the PACIFY trial [21]. Morphine reduces absorption and impairs P2Y12 inhibition for all P2Y12 agents $[21,28,29]$. Second, the potential cost savings of avoiding unnecessary fentanyl use is certainly important in this era of cost containment. Finally, and most importantly, the ever-increasing opioid epidemic is a major US health crisis with more than 42,000 Americans dying of opioid overdose each year $[11,12]$. As previously mentioned, the risk for dependence occurs not only with increasing the frequency of exposure but even with a single exposure to opioid medications $[14,15]$. For opioid-naïve patients, clinicians should strive to keep them opioid-naïve [30]. We believe that these facts present a compelling argument for limiting the use narcotics for sedation during routine elective CC.

4.1. Limitations. The primary limitations of our study include smaller sample size, single center, and single-blinded randomization. Other limitations involving the study design include patient reporting on a questionnaire, crossover allowance from midazolam monotherapy to midazolam and fentanyl, and more females in the midazolam plus fentanyl group. These limitations are minimized by the outcomes 


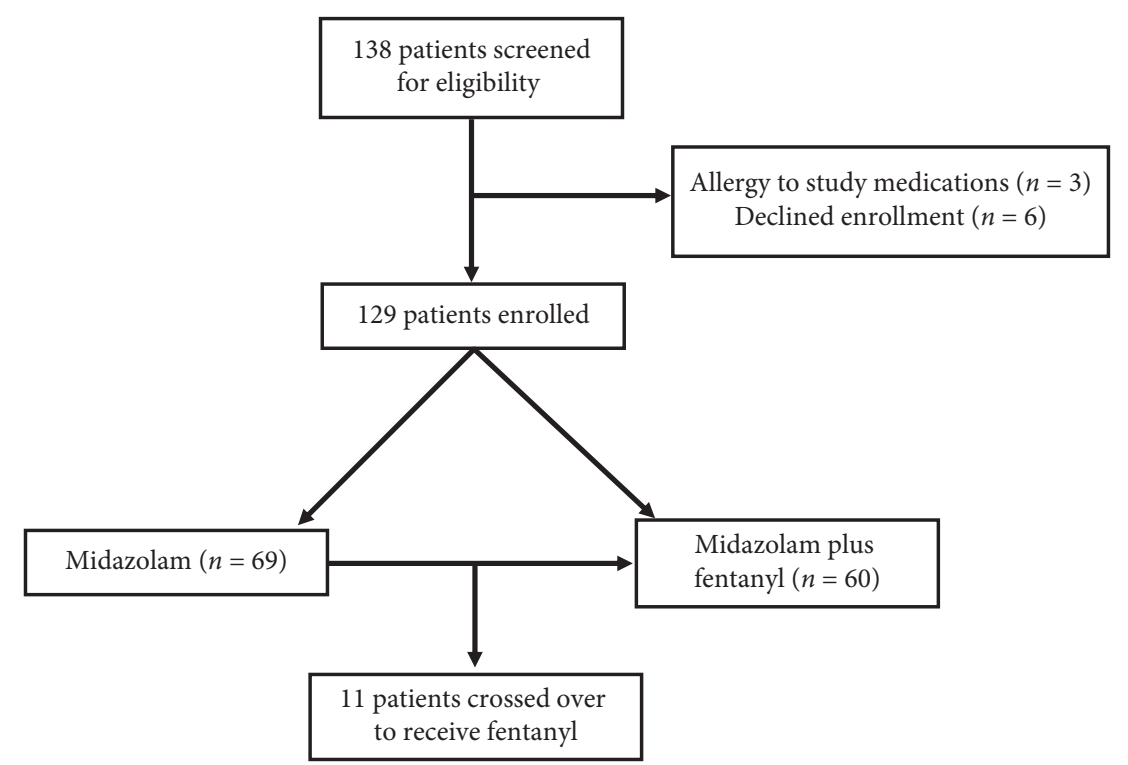

FIGURE 1: Flowchart of patient enrollment.

TABLE 1: Baseline characteristics by group.

\begin{tabular}{|c|c|c|c|}
\hline Variable & $\begin{array}{c}\mathrm{M} \\
N=69\end{array}$ & $\begin{array}{c}\mathrm{M}+\mathrm{F} \\
N=60\end{array}$ & $P$ value \\
\hline Age* $^{*}$ & $66.17(10.18)$ & $63.87(12.70)$ & 0.50 \\
\hline BMI* & $30.03(6.86)$ & $32.03(6.42)$ & 0.09 \\
\hline Gender (female), $n(\%)^{* *}$ & $15(21.74 \%)$ & $26(43.33 \%)$ & $0.008^{* * *}$ \\
\hline $\mathrm{CAD}^{* *}$ & $38(55.07 \%)$ & $27(45 \%)$ & 0.44 \\
\hline $\mathrm{CHF}^{* *}$ & $10(14.49 \%)$ & $6(10 \%)$ & 0.44 \\
\hline $\mathrm{HLD}^{* *}$ & $51(73.91 \%)$ & $40(66.67 \%)$ & 0.37 \\
\hline $\mathrm{DM}^{* *}$ & $17(24.64 \%)$ & $19(31.67 \%)$ & 0.38 \\
\hline $\mathrm{PVD}^{* *}$ & $5(7.25 \%)$ & $3(5 \%)$ & 0.60 \\
\hline $\mathrm{HTN}^{* *}$ & $55(79.71 \%)$ & $49(81.67 \%)$ & 0.78 \\
\hline Tobacco use $e^{* *}$ & $23(33.33 \%)$ & $13(21.67 \%)$ & 0.14 \\
\hline $\mathrm{CKD}^{* *}$ & $8(11.59 \%)$ & $4(6.67 \%)$ & 0.34 \\
\hline $\mathrm{UA}^{* *}$ & $2(2.90 \%)$ & $1(1.67 \%)$ & 0.65 \\
\hline Chronic pain ${ }^{* *}$ & $6(8.70 \%)$ & $6(10 \%)$ & 0.80 \\
\hline
\end{tabular}

${ }^{*}$ Values are mean (standard deviation). ${ }^{* *}$ Values are frequency (percentage). ${ }^{* * *}, P<0.05 . \mathrm{M}$, midazolam group; $\mathrm{M}+\mathrm{F}$, midazolam plus the fentanyl group.

TABLE 2: Comparison of independent groups on primary and secondary endpoints.

\begin{tabular}{|c|c|c|c|c|}
\hline Outcome & $\begin{array}{c}\mathrm{M} \\
N=69\end{array}$ & $\begin{array}{l}\mathrm{M}+\mathrm{F} \\
N=60\end{array}$ & Mean difference (95\% CI) & $P$ value \\
\hline Pain during procedure, $n(\%)^{* *}$ & $18(26.09 \%)$ & $17(28.33 \%)$ & - & 0.78 \\
\hline Pain severity $(1-10)^{*}$ & $1.1(2.0)$ & $1.1(2.3)$ & $-0.25(-0.77-0.72)$ & 0.95 \\
\hline Premidazolam $(\mathrm{mg})^{*}$ & $1.73(1.02)$ & $1.59(0.96)$ & $0.14(-0.21-0.49)$ & 0.43 \\
\hline Prefentanyl $(\mathrm{mg})^{*}$ & $0(0)$ & $50(25)$ & - & $0^{\dagger}$ \\
\hline Total midazolam (mg)* & $3.71(2.02)$ & $3.43(1.93)$ & $0.28(-0.42-0.97)$ & 0.43 \\
\hline Total fentanyl (mg)* & $0(0)$ & $75(50)$ & - & $0^{\dagger}$ \\
\hline Total procedure time (mins)* & $41.88(34.40)$ & $38.62(26.19)$ & $3.27(-7.51-14.05)$ & 0.55 \\
\hline
\end{tabular}

${ }^{*}$ Values are mean (standard deviation). ${ }^{* *}$ Values are frequency (percentage). ${ }^{\dagger} \mathrm{P}<0.05$. M, midazolam group; $\mathrm{M}+\mathrm{F}$, midazolam plus fentanyl group. ${ }^{\dagger} P$ value could not be calculated as fentanyl was not provided in the midazolam only group.

observed. Assessment of pain relied upon patient reporting postprocedure, which is subjective, and each patient may have a different pain tolerance. Eventhough our study design allowed for crossover, patients in the midazolam group rarely crossed over to receive fentanyl for sedation $(17 \%$, $n=12$ ). 


\section{Conclusion}

Midazolam monotherapy produces similar pain perception compared to a combination of midazolam and fentanyl during sedation during cardiac catheterization. The combination of midazolam and fentanyl has historically been the standard regimen for sedation in the catheterization lab. However, it may be important to consider midazolam monotherapy as an alternative. We believe a larger, randomized multicenter study is needed to further validate our findings. This is an important issue for multiple reasons including fentanyl's documented inhibition of absorption and efficacy of P2Y12 agents, potential cost savings of reducing fentanyl use, and reducing potential for opioid addiction. This study suggests that midazolam monotherapy is a reasonable option for sedation for cardiac catheterization.

\section{Data Availability}

The data used to support the findings of this study are available from the corresponding author upon request.

\section{Conflicts of Interest}

The authors declare that they have no conflicts of interest.

\section{Authors' Contributions}

All authors contributed evenly to the creation of this manuscript and are in agreement with the current version of the manuscript.

\section{References}

[1] S. S. Naidu, H. D. Aronow, and L. C. Box, "SCAI expert consensus statement: 2016 best practices in the cardiac catheterization laboratory: (endorsed by the cardiological society of India, and sociedad Latino Americana de Cardiologia intervencionista; affirmation of value by the Canadian Association of interventional cardiology-Association canadienne de cardiologie d'intervention)," Catheterization and Cardiovascular Interventions: Official Journal of the Society for Cardiac Angiography \& Interventions, vol. 88, no. 3, pp. 407-423, 2016.

[2] D. A. Leffler, B. Bukoye, M. Sawhney et al., "Development and validation of the PROcedural Sedation Assessment Survey (PROSAS) for assessment of procedural sedation quality," Gastrointestinal Endoscopy, vol. 81, no. 1, pp. 194-203.e1, 2015.

[3] American Society of Anesthesiologists Task Force on Sedation and Analgesia by Non-Anesthesiologists, "Practice guidelines for sedation and analgesia by non-anesthesiologists," Anesthesiology, vol. 96, no. 4, pp. 1004-1017, 2002.

[4] R. T. Pachulski, D. C. Adkins, and H. Mirza, "Conscious sedation with intermittent midazolam and fentanyl in electrophysiology procedures," Journal of Interventional Cardiology, vol. 14, no. 2, pp. 143-146, 2001.

[5] A. Natale, M. M. Kearney, M. J. Brandon et al., "Safety of nurse-administered deep sedation for defibrillator implantation in the electrophysiology laboratory," Journal of Cardiovascular Electrophysiology, vol. 7, no. 4, pp. 301-306, 1996.
[6] S. P. Thomas, J. Thakkar, P. Kovoor, A. Thiagalingam, and D. L. Ross, "Sedation for electrophysiological procedures," Pacing and Clinical Electrophysiology, vol. 37, no. 6, pp. 781-790, 2014.

[7] B. D. Gaitan, T. L. Trentman, S. L. Fassett, M. J. T. ueller, and G. T. Altemose, "Sedation and analgesia in the cardiac electrophysiology laboratory: a national survey of electrophysiologists investigating the who, how, and why?" Journal of Cardiothoracic and Vascular Anesthesia, vol. 25, no. 4, pp. 647-659, 2011.

[8] S. Lavi, S. S. Jolly, D. Bainbridge, F. Manji, V. Randhawa, and R. Lavi, "Sedation, analgesia, and anaesthesia variability in laboratory-based cardiac procedures: an international survey," Canadian Journal of Cardiology, vol. 30, no. 6, pp. 627-633, 2014.

[9] D. M. Phillips, "JCAHO pain management standards are unveiled. Joint Commission on Accreditation of Healthcare Organizations," JAMA, vol. 284, no. 4, pp. 428-429, 2000.

[10] B. A. Chidgey, K. L. McGinigle, and P. P. McNaull, "When a vital sign leads a country astray-the opioid epidemic," JAMA Surgery, vol. 154, no. 11, pp. 987-988, 2019.

[11] N. D. Volkow, E. B. Jones, E. B. Einstein, and E. M. Wargo, "Prevention and treatment of opioid misuse and addiction: a Review," JAMA Psychiatry, vol. 76, no. 2, pp. 208-216, 2019.

[12] K. T. Brady, J. L. McCauley, and S. E. Back, "Prescription opioid misuse, abuse, and treatment in the United States: an update," The American Journal of Psychiatry, vol. 173, no. 1, pp. 18-26, 2016.

[13] Q. Chen, M. R. Larochelle, D. T. Weaver et al., "Prevention of prescription opioid misuse and projected overdose deaths in the United States," JAMA Network Open, vol. 2, no. 2, Article ID e187621, 2019.

[14] A. C. Harris and J. C. Gewirtz, "Acute opioid dependence: characterizing the early adaptations underlying drug withdrawal," Psychopharmacology, vol. 178, no. 4, pp. 353-366, 2005.

[15] A. Shah, C. J. Hayes, and B. C. Martin, "Characteristics of initial prescription episodes and likelihood of long-term opioid use-United States, 2006-2015," Morbidity and Mortality Weekly Report, vol. 66, pp. 265-269, 2017.

[16] J. A. Hoppe, H. Kim, and K. Heard, "Association of emergency department opioid initiation with recurrent opioid use," Annals of Emergency Medicine, vol. 65, no. 5, pp. 493.e4-499.e4, 2015.

[17] M. K. Delgado, Y. Huang, Z. Meisel et al., "National variation in opioid prescribing and risk of prolonged use for opioidnaïve patients treated in the emergency department for ankle sprains," Annals of Emergency Medicine, vol. 72, no. 4, pp. 389-400.e1, 2018.

[18] R. Shah, A. J. Kirtane, and J. W. McEvoy, "Opiate use in the cath lab,” European Heart Journal, vol. 39, no. 8, pp. 642-645, 2018.

[19] P. T. O’Gara, F. G. Kushner, D. D. Ascheim et al., “ACCF/ AHA guideline for the management of ST-elevation myocardial infarction: a report of the American college of cardiology foundation/American heart association task force on practice guidelines," Circulation, vol. 127, no. 4, pp. e362-e425, 2013.

[20] E. A. Amsterdam, N. K. Wenger, R. G. Brindis et al., "AHA/ ACC guideline for the management of patients with non-STelevation acute coronary syndromes: a report of the American college of cardiology/American heart association task force on practice guidelines," Journal of the American College of Cardiology, vol. 64, no. 24, pp. e139-e228, 2014. 
[21] J. W. McEvoy, K. Ibrahim, T. S. Kickler et al., "Effect of intravenous fentanyl on ticagrelor absorption and platelet inhibition among patients undergoing percutaneous coronary intervention: the PACIFY randomized clinical trial (platelet aggregation with ticagrelor inhibition and fentanyl)," Circulation, vol. 137, no. 3, pp. 307-309, 2018.

[22] S. Baris, D. Karakaya, R. Aykent, K. Kirdar, O. Sagkan, and A. Tür, "Comparison of midazolam with or without fentanyl for conscious sedation and hemodynamics in coronary angiography," The Canadian journal of cardiology, vol. 17, no. 3 , pp. 277-281, 2001.

[23] J. Woodhead, S. A. Harding, M. Simmonds, S. Dee, and K. McBride-Henry, "Premedication for cardiac catheterization and percutaneous coronary intervention: does it increase vascular access site complications?" Journal of Cardiovascular Nursing, vol. 22, no. 6, pp. 466-471, 2007.

[24] A. Conway, J. Rolley, and J. R. Sutherland, "Midazolam for sedation before procedures," Cochrane Library: Cochrane Reviews, vol. 2016, no. 5, Article ID CD009491, 2016.

[25] S. Deftereos, G. Giannopoulos, K. Raisakis et al., "Moderate procedural sedation and opioid analgesia during transradial coronary interventions to prevent spasm: a prospective randomized study," JACC: Cardiovascular Interventions, vol. 6, no. 3, pp. 267-273, 2013.

[26] A. Mall, T. A. Girton, K. Yardley et al., "Understanding the patient experience of pain and discomfort during cardiac catheterization," Catheterization and Cardiovascular Interventions, vol. 95, no. 7, pp. E196-E200, 2020.

[27] C. Dal Piva, E. Vaz, M. A. Moraes et al., "Discomfort reported by patients after cardiac catheterization using the formal or radial approaches," Revista Brasileira Dr Cardiologia Invasiva, vol. 22, no. 1, pp. 36-40, 2014.

[28] E.-L. Hobl, T. Stimpfl, and J. Ebner, "Morphine decreases clopidogrel concentrations and effects: a randomized, doubleblind, placebo-controlled trial," Journal of the American College of Cardiology, vol. 63, no. 7, pp. 630-635, 2014.

[29] J. Kubica, P. Adamski, M. Ostrowska et al., "Morphine delays and attenuates ticagrelor exposure and action in patients with myocardial infarction: the randomized, double-blind, placebo-controlled IMPRESSION trial," European Heart Journal, vol. 37, no. 3, pp. 245-252, 2016.

[30] K. M. Babu, J. Brent, and D. N. Juurlink, "Prevention of opioid overdose," The New England Journal of Medicine, vol. 380, no. 23, pp. 2246-2255, 2019. 